\title{
Fast DRR Generation for 2D/3D Registration
}

\author{
W. Birkfellner ${ }^{1}$, R. Seemann ${ }^{1}$, M. Figl ${ }^{1}$, J. Hummel ${ }^{1,2}$, C. Ede ${ }^{1}$, P. Homolka $^{1}$, \\ X. Yang ${ }^{3}$, P. Niederer ${ }^{3}$, and H. Bergmann ${ }^{1,2}$ \\ 1 Center for Biomedical Engineering and Physics, Medical University Vienna, Austria \\ wolfgang. birkfellner@meduniwien.ac.at \\ www.bmtp.akh-wien.ac .at/people/birkwo1 \\ ${ }^{2}$ Ludwig-Boltzmann Insitute of Nuclear Medicine, Vienna \\ 3 Department of Biomedical Engineering, Swiss federal institute of Technology, \\ Zurich, Switzerland
}

\begin{abstract}
We present a simple and rapid method for generation of perspective digitally rendered radiographs (DRR) for $2 \mathrm{D} / 3 \mathrm{D}$ registration based on splat rendering. Suppression of discretization artefacts by means of computation of Gaussian footprints - which is a considerable computational burden in classical splat rendering - is replaced by stochastic motion of either the voxels in the volume to be rendered, or by simulation of a X-ray tube focal spot of finite size. The result is a simple and fast perspective rendering algorithm using only a small subset of voxels. Our method generates slightly blurred DRRs suitable for registration purposes at framerates of approximately $10 \mathrm{~Hz}$ when rendering volume images with a size of $30 \mathrm{MB}$ on a standard PC.
\end{abstract}

\section{Introduction}

Generation of simulated X-ray images or digitally rendered radiographs (DRR) is a key technology in 2D/3D registration for image guided therapy (IGT) 123 . and patient alignment in radiation oncology. In general, a DRR is a simulated X-ray image derived from a computed tomography (CT) volume by simulating the attenuation of virtual X-rays. Therefore a DRR is a perspective volume rendering. Raycasting, the computation of line integrals along selected beams emerging from the viewpoint, appears to be the logical rendering algorithm to fulfill this task. From a practical perspective, this approach is barely feasible. In $2 \mathrm{D} / 3 \mathrm{D}$ registration patient alignment is achieved by iteratively solving an optimization problem in 6 degrees-of-freedom (dof). During the optimization, the CT-volume undergoes rigid-body transformations where every dof is varied until an optimum match between DRR and X-ray image is achieved. Due to the complexity of the registration task a considerable number of DRRs have to be computed, and the time required for registration is closely related to the efficiency of DRR generation.

Fast perspective volume rendering for DRR generation is therefore a crucial issue for $2 \mathrm{D} / 3 \mathrm{D}$ registration. Several alternatives to conventional raycasting such as lightfield- 4] or shear-warp rendering [5] were proposed in the past. In this 
paper, we present a variation of splat rendering [6] - the direct projection of single voxels to the imaging plane - as a fast and simple alternative to these rendering algorithms. The main advantage of splatting lies in the fact that only voxels above a certain threshold are used for DRR generation; this can reduce the number of voxels to be visited during the rendering process significantly. Typically, a DRR of an abdominal CT scan uses less that $10 \%$ of the voxels for splat rendering of bony tissue. Therefore direct projection of voxels easily outperforms methods such as discrete raycasting by a factor of 10 - 20 depending on the minimum Hounsfield density to be rendered. The inherent problem of direct voxel projection is the massive decrease in image quality due to aliasing artefacts. The original splatting algorithm overcomes this problem by calculation of a 3D Gaussian kernel projected onto the image plane providing a discrete point spread function. For orthogonal projection, these so-called footprints can be computed in a preprocessing step. Unfortunately, this is not feasible for perspective splat rendering. As a result of applying antialiasing techniques, time requirements again increase considerable since for each voxel position in the CT volume, the projection of a discrete Gaussian 3D-kernel has to be computed as well. The initial advantage of the splatting algorithm over raycasting - its shorter execution cycle - is actually reversed by this additional task.

In this paper, we present a simple and efficient method for antialiasing of splat - rendered DRRs avoiding the computation of footprints. DRRs from splat rendering and raycasting are compared, and time requirements for DRR - generation were recorded.

\section{Materials and Methods}

The basic idea of splatting for generating a DRR is simple. Since the exponential weakening of the X-ray beam during body passage - which derives from the general law of linear attenuation [7, p. $59 \mathrm{ff}$ - can be taken into account on the projected image by logarithmization, a perspective summed voxel rendering is an acceptable volume rendering method for this purpose. Thus, every voxel can be projected by an algebraic perspective projection operator $P$ (actually a $4 \times 4$ matrix). A setup where the focal spot is located on the z-axis and the imaging plane lies in the $\mathrm{x}-\mathrm{y}$ plane is, for instance, a good starting point since it shows some favourable symmetry properties for registration [3].

Motion of the CT data set in this coordinate system is given by a $4 \times 4$ matrix $V$ which is composed out of a $3 \times 3$ rotation matrix and a translation vector. Details on both operators can be found in the literature, e. g. [13].

The contribution of each voxel can be computed by applying these operators to every voxel position $\boldsymbol{x}$ in the CT-volume;

$$
\boldsymbol{x}_{p}=P V \boldsymbol{x}
$$

resulting in coordinates $\boldsymbol{x}_{p}$ on the image plane. The HU of the voxel at location $\boldsymbol{x}$ is added to the actual grayscale value of the pixel located closest to $\boldsymbol{x}_{p}$. After applying eq. 1 to every voxel and integrating the projected density values, a 
summed voxel rendering of the CT-volume is the result. Discretization artefacts are inevitable when using this technique.

The method of directly projecting single voxel densities onto the projection plane also reveals the inherent advantage of splatting. Since the ordering of voxels relative to each other is of no importance, one can remove all voxels not contributing to the DRR. In the case of $2 \mathrm{D} / 3 \mathrm{D}$ registration, this is usually the CT volume content filled with soft tissue and air. After selecting a minimum and maximum threshold for voxels to be rendered, a single preprocessing step allows for reordering the volume.

It should be stated that eq. 1 can easily be generalized to positions of the $\mathrm{X}$-ray tube different to the geometry resulting in the projection operator $P$. As long as the projection plane remains normal to the central beam of the X-ray tube (for instance in a C-arm), a rigid body transform $V^{*}$ similar to the matrix $V$ can be used to model motion of the virtual tube focus. The projection operator $P$ is moved to $P^{*}$ by computing

$$
P^{*}=V^{*} P
$$

As said before the classical splatting technique overcomes the aliasing problem by means of projecting a Gaussian kernel for every voxel position. While the generation of the lookup table containing the splats is simply a preprocessing step in orthogonal projection, this is not the case for perspective projection. On the other hand, projecting such a kernel for every voxel position is a very time-consuming task. The quality of the antialiasing depends on the size of the Gaussian kernel. Taking into account that computation of a $3 \times 3 \times 3$ kernel requires 27 projections for a single footprint, it is evident that rendering times increase considerably, and that kernels with even larger size are definitely out of discussion. As an alternative, we have tested two methods where artefact suppression is achieved by stochastic motion of various parameters in the splatting process. These methods are a.) Gaussian motion of voxels and b.) Gaussian motion of the viewpoint.

The first method resembles a stochastic variant of the original splatting method; in this case, the projected voxel value is not spread on the projection plane by a footprint but by a stochastic Gaussian displacement of voxel positions $\boldsymbol{x}$ in a small range. For each voxel position $\boldsymbol{x}$, a $3 \times 1$ Gaussian random vector $\boldsymbol{u}$ with standard deviation $\sigma$ and mean 0 is computed using the Box-Muller method. Eq. 1 becomes

$$
\boldsymbol{x}_{p}=P V(\boldsymbol{x}+\boldsymbol{u})
$$

The standard deviation $\sigma$ of the Gaussian distribution was chosen in such a manner that $3 \sigma$ approximately equals the voxel size of the volume used.

The second approach is slightly more sophisticated and has the additional appeal of modelling the physics of X-ray generation. To some extent, it resembles methods for rendering of soft shadows in computer graphics. In general, the focal spot of an X-ray tube is not a mathematical point but an area approximately 2.0 $\mathrm{mm}$ in diameter [7, p. 42. This behaviour is simulated by stochastic translation of 
the projector $P$. parallel to the $\mathrm{x}-\mathrm{y}$ plane. An additional benefit of this approach is the fact that only two instead of three random numbers have to be computed, as opposed to the first approach. In detail, a $3 \times 1$ Gaussian random vector $\boldsymbol{v}=\left(v_{0}, v_{1}, 0\right)^{T}$ is computed. Again, mean of the Gaussian distribution is 0 and standard deviation $\sigma$ is chosen to be $\sigma=\frac{1}{3} D_{f s}$ where $D_{f s}$ is the diameter of the focal spot to be simulated. This displacement is applied to the projection operator $P^{*}$ (see eq. 2) by computing

$$
P^{\prime}=V^{*}\left(\begin{array}{cccc}
1 & 0 & 0 & v_{0} \\
0 & 1 & 0 & v_{1} \\
0 & 0 & 1 & 0 \\
0 & 0 & 0 & 1
\end{array}\right) P
$$

Here, the projection operator $P$ is configured in such a manner that it splats the voxels to the $\mathrm{x}-\mathrm{y}$ plane and the viewpoint is located at the z-axis. Applying $P^{\prime}$ instead of $P$ or $P^{*}$ respectively to the volumes voxel positions (eq. 1) results in geometric unsharpness as caused by a focal spot of finite size. We refer to both methods - the Gaussian distortion of voxel positions within the volume and the Gaussian motion of the virtual focal spot of the projector $P$ as 'wobbled splatting' since the antialiasing effect of footprint evaluation as in the classical splatting method is replaced by a random distortion of some splatting parameters.

A small application named SPLATTER for generation of DRRs was written in $\mathrm{C}++$ using the Qt 3.0 graphical user interface toolkit on standard Pentium IV with $2.8 \mathrm{GHz}$ under SuSE Linux 9.0. Additional image processing functionality was implemented using AVW 6.0 library (Biomedical Imaging Resource, Mayo Clinic, Rochester/MN). The Box-Muller method for generation of random numbers with a Gaussian distribution was taken form the GNU scientific library GSL 1.4 (Free Software Foundation, Boston/MA). All illustrations in this paper were generated using SPLATTER. AVW was provided courtesy of Dr. R. A. Robb, Biomedical Imaging Resource, Mayo Clinic, Rochester/MN.

For testing the various antialiasing methods, three CT volume scans were used. First, a clinical pediatric abdominal scan acquired with a Siemens Sensation CT (Siemens AG, Erlangen, Germany) with an original resolution of $512 \times 512 \times 151$ voxels was used. Since all scans were resampled using linear interpolation to $(1 \mathrm{~mm})^{3}$ voxels, the scan used for rendering had $237 \times 237 \times 302$ voxels and a size of $32.35 \mathrm{MB}$. It is to be noted that resampling to cubic voxels is performed for the sake of simplying the splatting algorithm, but it is not a necessity [6]. Second, a scan of a pelvis phantom interpolated to $290 \times 205 \times 200$ voxels and a size of $22.67 \mathrm{MB}$ was used. Finally, a clinical scan of mandible $(172 \times 172 \times 72$ voxels, $4.06 \mathrm{MB})$ was used. The latter two scans were acquired using a Philips Mx 8000 IDT scanner (Philips AG, Best, The Netherlands).

Evaluation was performed by applying various antialiasing techniques on DRRs rendered with an image size of $256 \times 256$ pixels and comparison of the outcome. A high-quality rendering using the interpolated raycasting routine from the AVW library was used as a gold standard. 


\section{Results}

Table 1 gives an overview of the visual outcome from the various antialiasing methods. Results from discrete and interpolated raycasting are also included. Voxel wobbling and focus wobbling combined with lowpass-filtering both provide good results. Figs. 1 and 2 show the effects of footprint evaluation and wobbling. The eight DRRs were rendered with a minmum threshold of $0 \mathrm{HU}$ and a maximum threshold of $3072 \mathrm{HU}$ from the pediatric abdomen scan. Fig. 1] shows considerable artefacts, which cannot be fully removed by multiplication with a footprint table generated from $3 \times 3 \times 3$ Gaussian kernels and subsequent lowpass-filtering (fig. 11).

Fig. 2 a shows a DRR generated using the same parameters as the DRRs in fig. 1 but rather than performing a footprint-evaluation, the image was generated with voxels being shifted by a Gaussian motion as given in eq. 3 with zero mean and standard deviation $\sigma=0.4 \mathrm{~mm}$. Fig. 2k was generated with static voxel positions; antialiasing is introduced by a Gaussian motion of the focal spot (or 'viewpoint') according to eq. 4 with zero mean and $\sigma=\frac{1}{3} \mathrm{~mm}$. While discretization artefacts are compensated for, Gaussian noise is clearly visible in both images. 2D lowpass filtering of the DRRs using a Gaussian $3 \times 3$ kernel compensate for this flaw (Figs. $2 \mathrm{~b}$ and $2 \mathrm{~d}$ ).

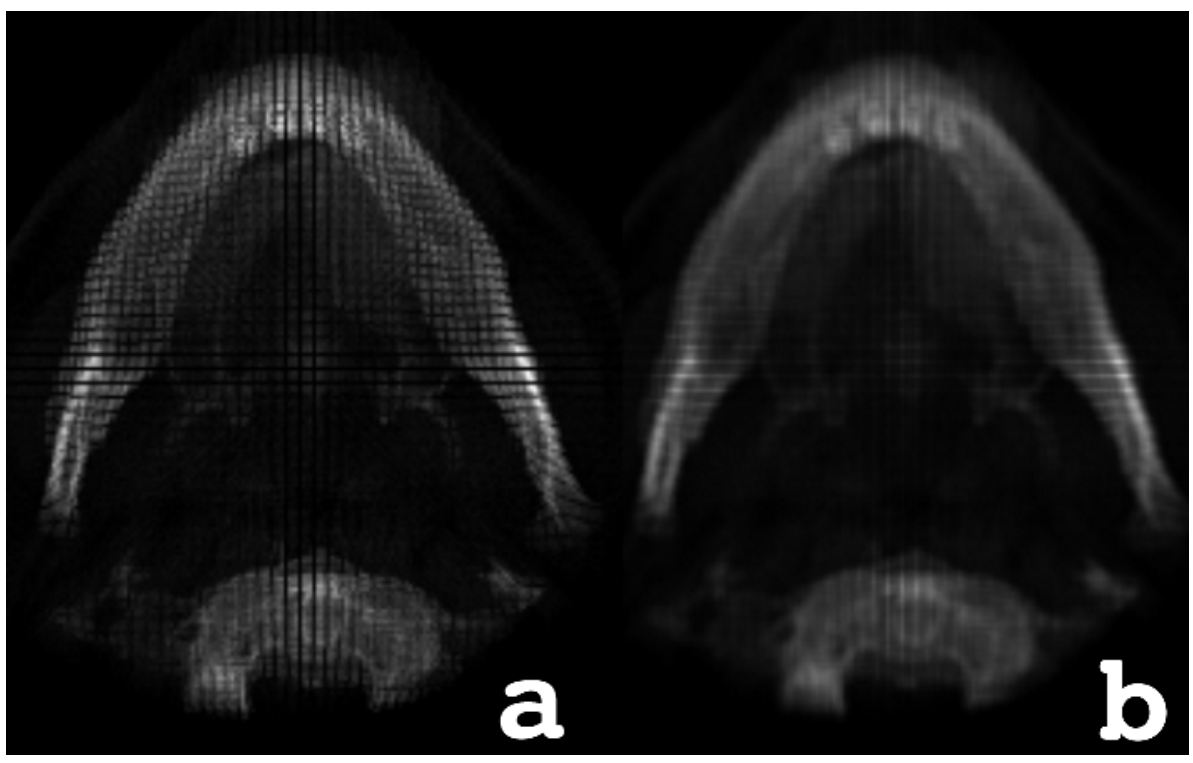

Fig. 1. Results of conventional splatting. The left image shows a direct projection of a mandible CT scan without further antialiasing measures. Discretization artefacts are clearly visible. The right image was splatted using Gaussian $3 \times 3$ kernels and lowpass filtering of the resulting DRR with a $3 \times 3$ kernel. 
Table 2 compares the amount of time required for DRR generation using splatting and raycasting combined with several antialiasing techniques. It is evident that the performance of splat rendering depends on the number of voxels used for DRR generation. Voxel wobbling and focus wobbling combined with subsequent lowpass filtering of the resulting DRR - the antialiasing techniques providing a sufficient result according to table 1- outperform interpolated raycasting typically by a factor of 20 when using a minimum threshold showing mainly bony tissue. Otherwise, splatting approximately requires the same amount of time as discrete raycasting. It was found that for the DRRs generated for this study, only 5 - $33 \%$ of the voxels had to be used.

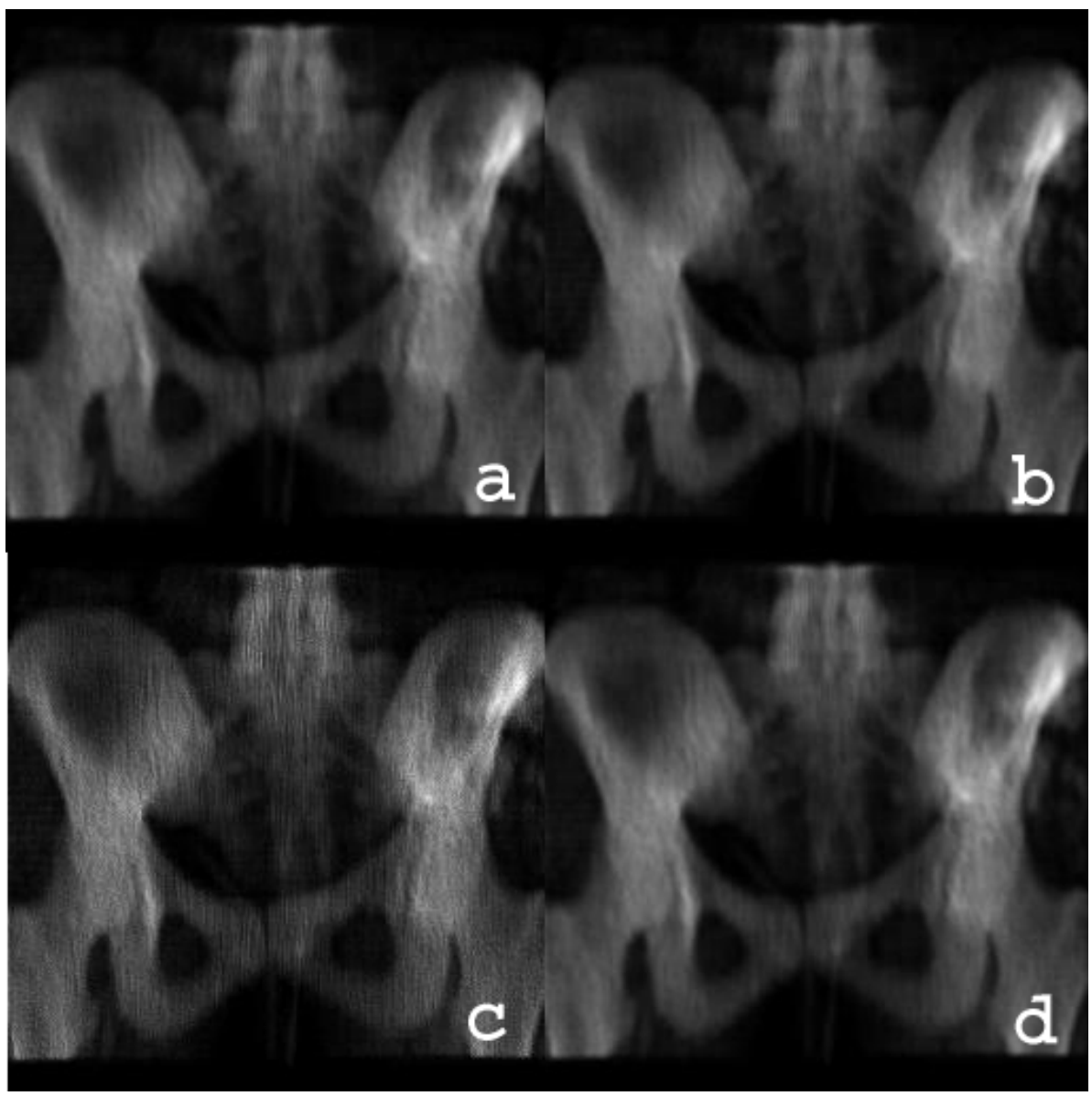

Fig. 2. Results of wobbled splatting. No footprint-table was generated, but either the voxel positions underwent displacement (a, voxel wobbling - voxels perform Gaussian movement with mean 0 and standard deviation $\sigma=0.4 \mathrm{~mm}$ ), or the focus position was blurred (c, focus wobbling - focal spot performs Gaussian movement with mean 0 and standard deviation $\sigma=0.33 \mathrm{~mm}$ ). b) shows a) after lowpass-filtering (again with a $3 \times 3$ kernel). d) shows c) after the same filtering procedure. 
Table 1. Visual effect of the various combinations of raycasting and antialiasing techniques. Rendering methods are splatting (SPL) and raycasting (RC). Antialiasing measures are lowpass filtering of the DRR using a $3 \times 3$ kernel (LP), evaluation of footprints from $3 \times 3 \times 3$ Gaussian footprints (GF), voxel wobbling (VW), focus wobbling (FW), and combinations thereof. Outcome was categorized to be heavily stricken with artefacts $(--)$, aliased $(-)$, fair $(\ldots)$, good $(+)$ and excellent $(++)$.

\begin{tabular}{|c|c|c|c|c|c|c|c|c|c|c|c|}
\hline $\begin{array}{c}\text { Renderer/ } \\
\text { Anti- } \\
\text { aliasing }\end{array}$ & SPL & $\begin{array}{c}\text { SPL/ } \\
\text { LP }\end{array}$ & $\begin{array}{c}\text { SPL/ } \\
\text { GF }\end{array}$ & $\begin{array}{l}\text { SPL/ } \\
\text { VW }\end{array}$ & $\begin{array}{l}\text { SPL/ } \\
\text { FW }\end{array}$ & $\begin{array}{l}\text { SPL/ } \\
\text { VW, } \\
\text { LP }\end{array}$ & $\begin{array}{l}\text { SPL/ } \\
\text { FW, } \\
\text { LP }\end{array}$ & $\begin{array}{l}\text { SPL/ } \\
\text { VW, } \\
\text { FW }\end{array}$ & $\begin{array}{c}\text { SPL/ } \\
\text { VW, } \\
\text { FW,LP }\end{array}$ & $\mathrm{RC}$ & $\begin{array}{c}\mathrm{RC} / \\
\text { inter- } \\
\text { polation }\end{array}$ \\
\hline $\begin{array}{c}\text { Visual } \\
\text { outcome }\end{array}$ & -- & - & - & & & + & + & & + & - & ++ \\
\hline
\end{tabular}

Table 2. Time required for DRR generation from three different CT scans given in seconds. Splat rendering (SPL) and raycasting $(\mathrm{RC})$ were used as rendering methods. Antialiasing techniques employed for splat rendering were lowpass filtering using a $3 \times 3$ Gaussian kernel (LP), prjoection using a footprint (point-spread function) calculated from a $3 \times 3 \times 3$ Gaussian kernel (GF), voxel wobbling (VW), focus wobbling (FW), and combiations. Finally, interpolated and discrete raycasting was used as well. Focus and voxel wobbling combined with $2 \mathrm{D}$ lowpass filtering - the preferrable antialiasing methods according to table 1- are printed in boldface.

\begin{tabular}{|c|c||c|c|c||c|c|c||c||c|c|}
\hline \multicolumn{3}{|c||}{ CT-Volume } & \multicolumn{3}{c||}{ Abdomen } & \multicolumn{3}{c||}{ Pelvis } & \multicolumn{3}{c|}{ Jaw } \\
\hline \hline \multicolumn{2}{|l||}{} & \multicolumn{2}{|c||}{ Min. Threshold } & \multicolumn{1}{|c|}{ Min. Threshold } & \multicolumn{2}{|c|}{ Min. Threshold } \\
\hline Render & Anti- & & & & & & & & & \\
method & aliasing & -205 & 0 & 205 & -980 & 0 & 350 & -1024 & 0 & 380 \\
\hline \hline SPL & - & 0.92 & 0.71 & 0.10 & 1.41 & 0.10 & 0.09 & 0.19 & 0.10 & 0.02 \\
SPL & LP & 0.94 & 0.71 & 0.10 & 1.40 & 0.11 & 0.12 & 0.20 & 0.10 & 0.02 \\
SPL & GF & 43.83 & 16.87 & 2.73 & 33.05 & 2.52 & 2.25 & 4.68 & 2.20 & 0.44 \\
SPL & VW & 1.24 & 0.83 & 0.12 & 1.65 & 0.13 & 0.11 & 0.23 & 0.11 & 0.02 \\
SPL & FW & 1.13 & 0.85 & 0.12 & 1.71 & 0.12 & 0.12 & 0.24 & 0.12 & 0.02 \\
SPL & VW, LP & $\mathbf{1 . 1 3}$ & $\mathbf{0 . 8 4}$ & $\mathbf{0 . 1 5}$ & $\mathbf{1 . 8 1}$ & $\mathbf{0 . 1 3}$ & $\mathbf{0 . 1 2}$ & $\mathbf{0 . 2 4}$ & $\mathbf{0 . 1 1}$ & $\mathbf{0 . 0 2}$ \\
SPL & FW, LP & $\mathbf{1 . 1 3}$ & $\mathbf{0 . 8 4}$ & $\mathbf{0 . 1 3}$ & $\mathbf{1 . 7 2}$ & $\mathbf{0 . 1 3}$ & $\mathbf{0 . 1 3}$ & $\mathbf{0 . 2 4}$ & $\mathbf{0 . 1 2}$ & $\mathbf{0 . 0 3}$ \\
SPL & VW, FW & 1.33 & 1.00 & 0.14 & 2.05 & 0.15 & 0.14 & 0.27 & 0.13 & 0.02 \\
SPL & VW, FW, LP & 1.40 & 1.00 & 0.15 & 2.03 & 0.17 & 0.14 & 0.28 & 0.14 & 0.03 \\
\hline RC & - & 1.63 & 1.68 & 1.64 & 1.16 & 1.07 & 1.12 & 0.08 & 0.09 & 0.08 \\
RC & interpolation & 6.15 & 2.75 & 2.33 & 7.17 & 1.82 & 1.77 & 0.42 & 0.30 & 0.13 \\
\hline \hline
\end{tabular}

\section{Discussion}

A fast DRR algorithm not requiring lengthy preprocessing is definitely desireable for clinical implementations of 2D/3D registration algorithms. For the particular case of iterative DRR generation for registration, splatting has the inherent advantage of its simplicity. The considerable time necessary for generating the footprint tables calls for an alternative solution for the reduction of aliasing artefacts. For this reason, footprint tables generated by Gaussian kernels with a 
dimension beyond $3 \times 3 \times 3$ were not included in this paper. As mentioned before, the reduction of detail due to antialiasing is not considered a severe drawback since measures similar to lowpass filtering have to be undertaken to avoid local minima in the optimization process [12]. Aliasing artefacts, on the other hand, cannot be tolerated since small structures with high intensity variations tend to introduce considerable misregistration for a number of $2 \mathrm{D} / 3 \mathrm{D}$ cost functions such as minimization of cross-correlation and algorithms based on image gradiente evaluation. The advantage of wobbled splatting compared to anti-aliasing by lowpass-filtering with larger kernels lies in the fact that structure and detail is conserved to some extent. In this paper we have shown that DRRs of bony structures (i. e. DRRs rendered with a minimum threshold above typical soft tissue radioopacity) can be rendered without visible artefacts at framerates of approximately $10 \mathrm{~Hz}$. This amounts to $5-7 \%$ of the computing time necessary for interpolated raycasting.

While comparing rendering algorithms applied to different volumes remains difficult, it has to be stated that this increase in computational efficiency compares well to the numbers reported in the literature. We conclude that wobbled splatting is comparable to these rendering algorithms in terms of efficiency; it provides an interesting alternative for generation of DRRs in iterative registration applications.

\section{References}

1. Lemieux L, Jagoe R, Fish DR, Kitchen ND, Thomas DG: A patient-to-computedtomography image registration method based on digitally reconstructed radiographs. Med Phys 21(11):1749-1760, (1994)

2. Penney GP, Weese J, Little JA, Desmedt P, Hill DL, Hawkes DJ: A comparison of similarity measures for use in 2-D-3-D medical image registration. IEEE Trans Med Imaging 17(4):586-595, (1998)

3. Birkfellner W, Wirth J, Burgstaller W, Baumann B, Staedele H, Hammer B, Gellrich NC, Jacob AL, Regazzoni P, Messmer P: A faster method for 3D/2D medical image registration-a simulation study. Phys Med Biol 48(16):2665-2679, (2003)

4. LaRose D: "Iterative X-Ray-CT Registration using Accelerated Volume Rendering", PhD thesis, Carnegie Mellon University, (2001)

5. Lacroute P, Levoy M: Fast volume rendering using a shear-warp factorization of the viewing transformation. SIGGRAPH'94:451-458, (1994)

6. Westover L: Footprint evaluation for volume rendering. SIGGRAPH'90:367-376, (1990).

7. Dendy PP, Heaton B: "Physics for Diagnostic Radiology", IOP Medical Science Series, (1999). 